\title{
FALL-BACK DISKS IN LONG AND SHORT GRBS
}

\author{
J. K. CANnIZzO ${ }^{1,2}$, E. TrojA ${ }^{3,4}$, AND N. Gehrels ${ }^{4}$ \\ Draft version October 24, 2018
}

\begin{abstract}
We present numerical time-dependent calculations for fall-back disks relevant to GRBs in which the disk of material surrounding the black hole $(\mathrm{BH})$ powering the GRB jet modulates the mass flow, and hence the strength of the jet. Given the initial existence of a small mass $\lesssim 10^{-4} M_{\odot}$ near the progenitor with a circularization radius $\sim 10^{10}-10^{11} \mathrm{~cm}$, an unavoidable consequence will be the formation of an "external disk" whose outer edge continually moves to larger radii due to angular momentum transport and lack of a confining torque. For long GRBs, if the mass distribution in the initial fall-back disk traces the progenitor envelope, then a radius $\sim 10^{11} \mathrm{~cm}$ gives a time scale $\sim 10^{4} \mathrm{~s}$ for the X-ray plateau. For late times $t>10^{7} \mathrm{~s}$ a steepening due to a cooling front in the disk may have observational support in GRB 060729. For short GRBs, one expects most of the mass initially to lie at small radii $<10^{8} \mathrm{~cm}$; however the presence of even a trace amount $\sim 10^{-9} M_{\odot}$ of high angular material can give a brief plateau in the light curve. By studying the plateaus in the X-ray decay of GRBs, which can last up to $\sim 10^{4} \mathrm{~s}$ after the prompt emission, Dainotti et al. find an apparent inverse relation between the X-ray luminosity at the end of the plateau and the duration of the plateau. We show that this relation may simply represent the fact that one is biased against detecting faint plateaus, and therefore preferentially sampling the more energetic GRBs. If, however, there were a standard reservoir in fall-back mass, our model can reproduce the inverse X-ray luminosity-duration relation. We emphasize that we do not address the very steep, initial decays immediately following the prompt emission, which have been modeled by Lindner et al. as fall-back of the progenitor core, and may entail the accretion of $\gtrsim 1 M_{\odot}$.
\end{abstract}

Subject headings: Accretion, accretion disks - Gamma ray burst: general - Gamma-ray burst: individual: GRB 060729, GRB 051221A

\section{INTRODUCTION}

The tentative pre-Swift hints that breaks occur in the longterm afterglow light curves simultaneously in different wavebands at $\sim 1-10 \mathrm{~d}$ (Frail et al. 2001) after the GRB have not been borne out by numerous detailed observations of Swift GRB afterglows in recent years (Ghisellini et al. 2007, Oates et al. 2007, Racusin et al. 2008, Liang et al. 2008). With Swift (Gehrels et al. 2004), the long-term $\left(t \gtrsim 10^{6} \mathrm{~s}\right) \mathrm{X}$-ray behavior has turned out to be surprisingly complex, exhibiting alternating steep and shallow slopes when plotted as log $F_{x}$ versus $\log (t-T 0)$, where $T 0$ is the trigger time for prompt BAT emission (Zhang et al. 2006; Nousek et al. 2006).

The traditional viewpoint that long-term fading of the GRB afterglow represents the deceleration and spreading of a relativistic jet (e.g., Sari, Piran, Halpern 1999, Frail et al. 2001) has given way to a much broader effort involving a diverse variety of hypotheses by which a central engine can be powered for a long time, including such scenarios as magnetars (Fan \& Xu 2006; Toma et al. 2007; Rowlinson et al. 2010), quark stars (Staff, Niebergal, \& Ouyed 2008), and long term accretion onto a BH formed during the collapsar or NS-NS merger (Kumar et al. 2008a, 2008b; Metzger et al. 2008a, $2008 \mathrm{~b})$. The accretion hypothesis entails exploring the possibility that the long-term decay of the X-ray flux is not due to the deceleration of baryonic ejecta, but rather a secular decrease in the rate of accretion powering the central engine, and therefore indirectly the jet (Kumar et al. 2008a, 2008b;

\footnotetext{
${ }^{1}$ CRESST and Astroparticle Physics Laboratory NASA/GSFC, Greenbelt, MD 20771; John.K.Cannizzo@ nasa.gov

${ }^{2}$ Department of Physics, University of Maryland, Baltimore County, 1000 Hilltop Circle, Baltimore, MD 21250

${ }^{3}$ NASA Postdoctoral Program Fellow

${ }^{4}$ Astroparticle Physics Laboratory NASA/GSFC, Greenbelt, MD 20771
}

Metzger et al. 2008a, 2008b, Cannizzo \& Gehrels 2009, hereafter CG09). For long GRBs (IGRBs), the early, steep rate of decay may be giving us information about the radial density distribution within the progenitor core (Kumar et al. 2008b), whereas the later decay may be governed by the outward expansion of the transient disk formed from the remnants of the progenitor (CG09). As regards short GRBs (sGRBs), even if a small amount of material $\left(\sim 10^{-5}-10^{-4} M_{\odot}\right)$ is expelled during the NS-NS merger and later accreted in a disk, that would be sufficient to power a bright afterglow, which may also be strongly influenced by the effects of r-process nucleosynthetic heating in the neutron rich material that becomes the disk (Metzger et al. 2010).

Zhang et al. (2006) present a schematic for the decaying GRB light curve as seen by the XRT on Swift. The decay is traditionally shown in $\log F-\log t$. There are four basic power-law decay $\left(F \propto t^{-\alpha}\right)$ regimes: (i) a steep decline following the prompt emission with $\alpha_{\mathrm{I}} \simeq 3$ out to $10^{2}-10^{3} \mathrm{~s}$, (ii) a plateau with $\alpha_{\mathrm{II}} \simeq 0.5$ out to $10^{3}-10^{4} \mathrm{~s}$, (iii) a steepening with $\alpha_{\mathrm{III}} \simeq 1.2$ out to $10^{4}-10^{5} \mathrm{~s}$, and (iv) a further steepening at late times (not always seen) with $\alpha_{\mathrm{IV}} \simeq 2$.

CG09 present a general analytical formalism to explain the different power law decays using a fall-back disk, where the variations in $\alpha$ could potentially be explained by different physics operating within the disk. The results of CG09 were purely analytical; in this work we present time dependent numerical calculations in order to examine in more detail the potential of the model, and we apply the results to XRT data for one lGRB and one sGRB, taking the best studied of each class. In Section 2 we review the Dainotti relation, an empirical relation involving the duration and luminosity of segment II, in Section 3 we present our detailed numerical model, in Section 4 we compare the model with observations for the 
1GRB 060729, the GRB with the longest observational time series in X-rays, in Section 5 we compare theory and data for the sGRB 051221A, the sGRB with the longest and most detailed XRT light curve, in Section 6 we revisit the Dainotti relation, in the context of our numerical results, and in Section 7 we discuss and summarize our results.

\section{THE DAINOTTI RELATION}

Dainotti et al. (2008, 2010) found an empirical relation between the duration of the X-ray plateau in the source frame $t_{\mathrm{II}}^{*}=t_{\mathrm{II}}(1+z)^{-1}$, and the X-ray luminosity at the end of the plateau, also corrected into the source frame, $L_{\mathrm{II}}^{*}$. Expressing their relation in the form $\log _{10} L_{\mathrm{II}}^{*}\left(\mathrm{erg} \mathrm{s}^{-1}\right)=$ $a+b \log _{10} t_{\mathrm{II}}^{*}(\mathrm{~s})$, Dainotti et al. (2010) find $a=51.1 \pm 1$ and $b=-1.1 \pm 0.3$. Their sample of IGRBs with small errors is defined by $u<4$, where $u \equiv\left[\left(\log \delta L_{\mathrm{II}}^{*}\right)^{2}+\left(\log \delta t_{\mathrm{II}}^{*}\right)^{2}\right]^{1 / 2}$, and $\log \delta L_{\mathrm{II}}^{*}$ and $\log \delta t_{\mathrm{II}}^{*}$ represent the logarithmic errors in $L_{\mathrm{II}}^{*}$ and $t_{\mathrm{II}}^{*}$, respectively. Using the same sample of 62 lGRBs with small errors taken from Dainotti et al. (2010), we find ${ }^{5}$ $a=50.5 \pm 0.4$ and $b=-0.92 \pm 0.1$, close to their results.

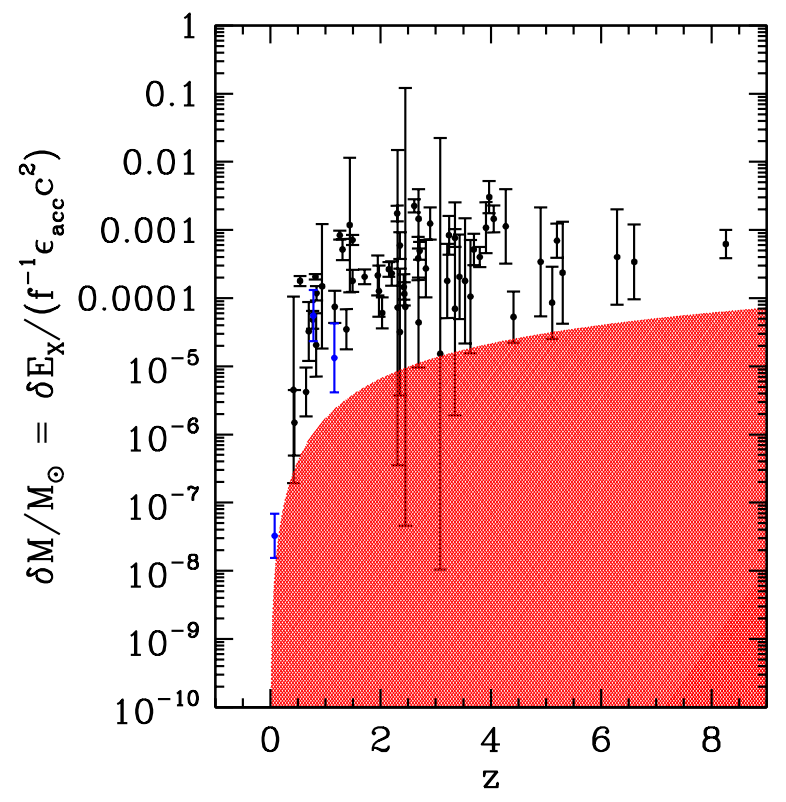

FIG. 1.- Inferred total accretion mass for the plateau + later decay phases of GRBs, using data from Dainotti et al. (2010) for their 62 well-constrained lGRBs. The hatched area (shown in red) indicates a putative limiting XRT detection flux level $f_{\mathrm{II}}=10^{-12} \mathrm{erg} \mathrm{cm}^{-1} \mathrm{~s}^{-1}$ (adopting a plateau duration $t_{\mathrm{II}}=10^{4} \mathrm{~s}$ ) for being able to study a plateau to sufficient accuracy that it would have been included in the Dainotti et al. sample of 62 GRBs with good statistics and known redshifts. (Three of the redshifts given by Dainotti et al., $z=0.08$ for $051109 \mathrm{~B}, z=0.78$ for 060202 , and $z=1.16$ for 07051B, are less secure than the others, i.e., based either on photometry or $\mathrm{X}$-ray spectroscopy, and have been indicated by blue symbols.) We adopt a beaming factor $f=1 / 300$ and a net efficiency for powering the X-ray flux $\epsilon_{\text {net }}=\epsilon_{\text {acc }} \epsilon_{X}=0.03$ to convert from X-ray fluence (i.e., total X-ray energy, after taking into account $4 \pi d_{L}^{2}$ ) to accreted mass.

If the inverse relation between $L_{\mathrm{II}}^{*}$ and $t_{\mathrm{II}}^{*}$ is physical, and

5 We employ a different method than Dainotti et al. (2010), a Monte Carlo technique in which $10^{6}$ data sets are created with $1 \sigma$ errors randomly either added or subtracted to each data point in both $x$ and $y$ directions. Thus for $N$ data points one could in principle have $N^{4}$ distinct data sets. For each data set, $a$ and $b$ values are calculated, and the final values for $a, \delta a, b$, and $\delta b$ are taken from the averages and standard deviations of the $10^{6}$ values. if long-term accretion is the correct explanation for the longterm X-ray light curve of GRBs, that would have implications for the amount of mass in the initial fall-back disk. To first order, the fact that $L_{\mathrm{II}}^{*} t_{\mathrm{II}}^{*}$ is constant would imply the accreted mass reservoir is constant. Willingale et al. (2007) present a simple formalism for integrating the X-ray flux on the plateau and subsequent $\alpha_{\text {III }} \simeq 1.3$ decay to obtain a total energy for segments II and III. We can convert this into a mass by making a few plausible assumptions about the energetic efficiencies (Krolik, Hawley, \& Hirose 2007), e.g., an X-ray afterglow beaming factor $f \simeq 10^{-3}-10^{-2}$, and an efficiency $\epsilon_{\text {net }} \simeq 0.01-0.1$ with which accretion onto the inner engine powers the observed X-rays, presumed to be created within the beamed jet. This overall accretion efficiency has two components, (1) the efficiency $\epsilon_{\text {acc }}$ with which accretion onto the $\mathrm{BH}$ powers the jet, where $\epsilon_{\mathrm{acc}} \simeq 0.4$ for the anticipated high spin BHs in GRBs, and (2) the efficiency $\epsilon_{X}$ with which the jet power is converted into $0.3-10 \mathrm{keV}$ X-rays that can be observed by XRT. Therefore $\epsilon_{\text {net }}=\epsilon_{\text {acc }} \epsilon_{X}$. We use eqns. [3] and [4] from Willingale et al. (2007) to obtain a total energy $\delta E_{X}$ and hence $\delta M$ derived from the X-ray fluence, for segments II and III. The results are shown in Figure 1. The hatched area is bounded from above by an estimate of the effective limiting XRT flux ${ }^{6}$ for being able to observe and characterize plateaus sufficiently well that they would satisfy $u<4$. There appears to be a selection effect giving rise to the Dainotti relation, namely that the flux detection limit for XRT prevents one from clearly observing and parameterizing faint plateaus at high $z$. The Dainotti relation, effectively equivalent to $L_{\mathrm{II}}^{*} t_{\mathrm{II}}^{*} \simeq$ constant, results from the sampling of GRBs primarily beyond $z \simeq 1.5$, for which faint plateaus are observationally biased against. Thus we are seeing basically a narrow strip corresponding to the upper end of a much broader distribution, which makes it appear that $L_{\mathrm{II}}^{*} t_{\mathrm{II}}^{*} \simeq$ constant. For $z \lesssim 1.5$ one sees curvature in the lower envelope of $\delta E_{X}$ values due to the strong dependence of the detection limit on $z$. Nevertheless, the fact that there appears to be a well-defined upper limit $\delta M \approx 10^{-4}-10^{-3} M_{\odot}$ is interesting: if one starts with $\mathrm{a} \sim 10 M_{\odot}$ progenitor and if accretion governs the long-term $\mathrm{X}$-ray light curve, this indicates that no more than a fraction $\sim 10^{-5}-10^{-4}$ of the progenitor mass survives the hypernova to form a fall-back disk (excluding segment I). Given the potential for strong outflows during the accretion process, this $\delta M$ should be viewed as a lower limit.

\section{ACCRETION DISK PHYSICS}

By writing the equations for mass continuity and angular momentum transport in cylindrical coordinates, assuming Keplerian rotation $\Omega_{K}^{2}=G M_{\mathrm{BH}} r^{-3}$ and integrating over the vertical thickness of the accretion disk, one arrives at an equation for the evolution of the surface density $\Sigma=2 \rho h$, where $\rho$ is the density and $h$ the disk semithickness (actually pressure scale height),

$$
\frac{\partial \Sigma}{\partial t}=\frac{3}{r} \frac{\partial}{\partial r}\left[r^{1 / 2} \frac{\partial}{\partial r}\left(\nu \Sigma r^{1 / 2}\right)\right] .
$$

${ }^{6}$ Our adopted XRT plateau detection level $10^{-12} \mathrm{erg} \mathrm{cm}^{-1} \mathrm{~s}^{-1}$ is unrelated to the nominal single pointing detection limit $\sim 10^{-14} \mathrm{erg} \mathrm{cm}^{-1}$ $\mathrm{s}^{-1}$, and in fact exceeds it considerably: In order to distinguish the different phases of the canonical light curve (i.e., plateau versus steep decay), one must take into account the effect of Swift orbital gaps, which places a much stronger constraint on the light curve characterization than simple flux detection. In addition, the presence of flares may further complicate the picture, but they usually are confined to $t \lesssim 10^{3} \mathrm{~s}$ and generally do not represent a major portion of the total energy budget. 
The kinematic viscosity coefficient

$$
\nu=\frac{2 \alpha_{\mathrm{SS}} P}{3 \Omega_{K} \rho},
$$

where $P$ is the pressure and $\alpha_{\mathrm{SS}}$ is the Shakura-Sunyaev parametrization of the angular momentum transport and heating (Shakura \& Sunyaev 1973). Guided by the current concordance between inferred values of $\alpha_{\mathrm{SS}} \simeq 0.1$ from dwarf nova outburst decays, both fast, thermal decays (Smak 1984) and slow, viscous decays (Cannizzo et al. 2010), and global 3D general relativistic magnetohydrodynamic (GRMHD) models of the magnetorotational instability (MRI) which also give $\alpha_{\mathrm{SS}} \simeq 0.1$ at large radii in the disk (McKinney \& Narayan $2007 \mathrm{ab}$ ), we set $\alpha_{\mathrm{SS}}=0.1$. Equation (1) is discretized following the method of Bath \& Pringle (1981), in which grid points are distributed as $r^{1 / 2}$. In addition, one has a thermal energy equation governing the temperature evolution,

$$
\frac{\partial T}{\partial t}=\frac{2(A-B+C+D)}{c_{p} \Sigma}-\frac{\mathcal{R} T}{\mu c_{p}} \frac{1}{r} \frac{\partial}{\partial r}\left(r v_{r}\right)-v_{r} \frac{\partial T}{\partial r},
$$

where the viscous heating $A=(9 / 8) \nu \Omega^{2} \Sigma$, the radiative cooling $B=\sigma T_{e}^{4}$, and $C$ and $D$ represent radial heat fluxes due to turbulent and radiative transport (Cannizzo et al. 2010). For the calculations we present in this work, the thermal equation only becomes of potential importance at very late times $>1 \mathrm{yr}$. In other words, $A=B$ to very good precision for most of the evolution, due to the fact that $\zeta \equiv$ $d \log T(\Sigma) / d \log \Sigma>0$. In other words, disks with $\zeta>0$ are viscously and thermally stable (Piran 1978), and therefore simple viscous evolution provides a satisfactory physical description.

After $\sim 1$ yr the surface density in the very outer disk drops below that associated with the hysteresis due to the transition between ionized and neutral gas, the source of the dwarf nova limit cycle mechanism. This launches a cooling front that propagates to smaller radii and rapidly shuts off the supply of gas to the inner disk, as the effective viscosity plummets. The dwarf nova limit cycle is an accretion disk instability in which the $T(\Sigma)$ relation exhibits a hysteresis at a temperature corresponding roughly to the peak in the Rosseland opacity curve - effectively an "S"-curve when plotted as $T$ versus $\Sigma$. It accounts for the outbursts seen in dwarf novae (Lasota 2001). The global manifestation of the instability is that material accumulates in the disk during quiescence and accretes onto the central star during outburst. The transition between the two states is mediated by the action of heating and cooling fronts. The cooling front we see at late times in our calculations are a result of the disk mass becoming so small that the transition from ionized to neutral gas is instigated. (A detailed discussion of the effects associated with the heating and cooling fronts is given in Cannizzo [1993, 1998].)

At early times and small radii the local disk accretion can be significantly super-Eddington, and in the thin disk formalism one would have $h / r>>1$, an inconsistency. Section 2.3.2 of CG09 contains scalings for the slim disk branch of solutions (Abramowicz et al. 1988) which we employ when $h / r>$ $(3 / 4)^{1 / 2}$ locally. Combining eqns. (24) and (26) of CG09 gives

$$
T(\Sigma)=1.56 \times 10^{5} \mathrm{~K} \mathrm{~m}_{\mathrm{BH}, 1}^{1 / 4} r_{11}^{-1 / 2} \Sigma^{1 / 4},
$$

where $m_{\mathrm{BH}, 1}=M_{\mathrm{BH}} /\left(10 M_{\odot}\right)$ and $r_{11}=r /\left(10^{11} \mathrm{~cm}\right)$. For completeness, we note that at early times and small radii, other types of disks are possible, such as neutrino-cooled accretion disks (e.g., Narayan, Piran, \& Kumar 2001; Kohri, Narayan, \& Piran 2005; Chen \& Beloborodov 2007). The controlling dynamics of the long term accretion rate is governed by the physical state of the outer disk, since that is where the slowest (i.e., controlling) time scale is. A complete physical modeling of the disk at small radii, including neutrino cooling and nucleosynthesis, is beyond the scope of this work, but also not necessary given in our primary interest, the long term accretion rate. At late times and large radii where $h / r<(3 / 4)^{1 / 2}$, we utilize the thin disk scalings from CG09. Combining eqns. (10) and (11) from CG09 gives

$$
T(\Sigma)=84.1 \mathrm{~K} \alpha_{\mathrm{SS},-1}^{1 / 3} m_{\mathrm{BH}, 1}^{1 / 6} r_{11}^{-1 / 2} \Sigma^{2 / 3},
$$

where $\alpha_{\mathrm{SS},-1}=\alpha_{\mathrm{SS}} / 0.1$. From a pragmatic standpoint, even though the use of a thin disk at small radii would be inconsistent because one would find $h / r>>1$, the use of a slim disk at small radii versus a thin disk has minimal effect on the light curve. This comes about because the controlling time scale in the problem comes from the largest disk radius. In fact, one could probably model the entire disk as a one-zone model, taken at the outer disk edge as was done by Metzger et al. (2008a) for NS-NS merger disks, and still get a reliable result.

The composition of the accretion disk is substantially different from solar. The $T(\Sigma)$ relation exhibits a weak inverse scaling with mean molecular weight $\mu$ for gas pressure and electron scattering opacity, $T \propto \mu^{-1 / 3}$ (Cannizzo \& Reiff 1992). The expected mixture of alpha elements yields $\mu \simeq 16$, rather than the value $\mu \simeq 0.65$ more generally relevant for solar composition material. Thus $T \propto \mu^{-1 / 3}$ brings about a decrease in $T(\Sigma)$ by a factor $\simeq 2.9$ at given surface density, and therefore $h / r$ by a factor $\simeq 1.7$, compared to solar composition material.

For completeness, we note that significant outflow may accompany accretion flows that are substantially superEddington. For computational expedience we only consider the slim disk in the super-Eddington limit, since the addition of outflows would introduce additional free parameters. However, given the strong potential for outflow, we stress that our accreted masses represent lower limits as to the amount of mass that may actually be accreted during segments II and III. Similarly, the inferred accreted masses shown in Figure 1 may significantly underestimate the true remnant masses left over after the collapsar event.

CG09 present analytical models for the afterglow light curves powered by fall-back disks. The main strength of model is the universal decay law $d \log L_{\text {acc }} / d \log t \simeq-1.3$ characterizing fall-back disks without the external, confining tidal torque of a companion star (Cannizzo, Lee, \& Goodman 1990). This law seems consistent with the late time decay of GRBs seen in X-rays. The early time decay, in particular the steep decay for $t \lesssim 10^{3} \mathrm{~s}$, following the prompt emission, is difficult to explain within the fall-back disk scenario and may be giving us information about the radial density profile within the progenitor (Kumar et al. 2008b). In this work we present results of time dependent numerical calculations using a general accretion disk code (Cannizzo et al. 2010), which uses the input physics detailed in CG09. The boundary conditions are as follows: (i) at the inner disk edge, taken to be $10^{7} \mathrm{~cm}$ for sGRBs and $10^{8} \mathrm{~cm}$ for lGRBs, matter is instantly removed as it arrives, while (ii) the outer grid point is placed at such a large radius, typically $10^{13} \mathrm{~cm}$, that during the course of the run the outer edge of the spreading fall-back 

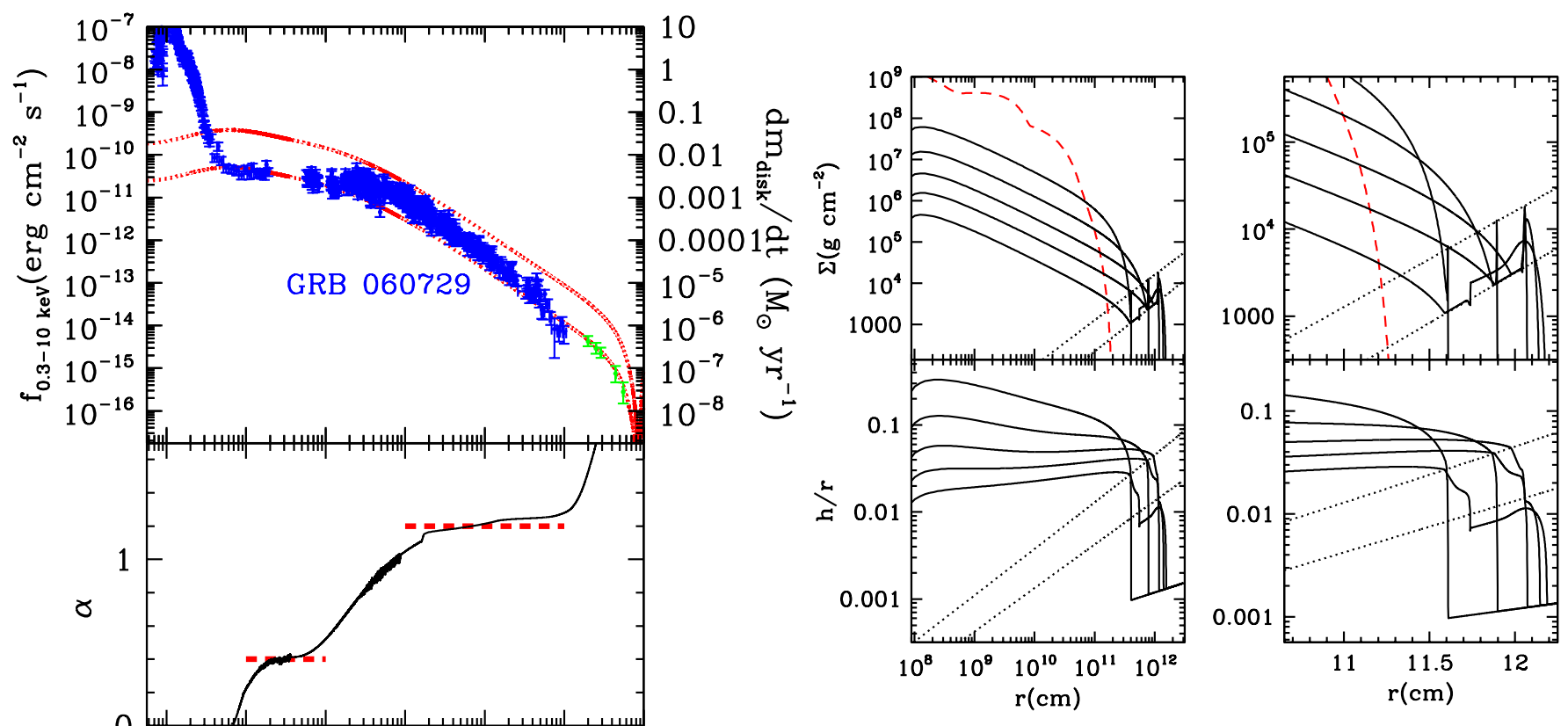

FIG. 3.- The disk evolution accompanying Figure 1. The upper panels show $\Sigma(r)$ (solid lines, top to bottom) at $t=5,25,100,300$, and $600 \mathrm{~d}$. The dashed line indicates the initial $\Sigma(r)$ distribution, and the parallel dotted lines indicate the critical values associated with the dwarf nova limit cycle which occurs at the transition between neutral and ionized gas. The lower panels show the evolution of $h / r$ corresponding to the same times as in the upper panels. curves (in red) to convert from rate of accretion onto the central engine to XRT fluxes are $f \epsilon_{\text {net }}^{-1}=8.8 \times 10^{-3}$ (upper) and $1.4 \times 10^{-2}$ (lower). The accretion rate values given on the right hand side axis correspond to an overall efficiency $f \epsilon_{\text {net }}^{-1}=4.9 \times 10^{-3}$. The five flux values at late times $10^{7} \mathrm{~s}$ $<t<10^{8} \mathrm{~s}$ (in green) come from Chandra observations (Grupe et al. 2010). The last data point lies at $t-T 0=642 \mathrm{~d}$. The initial profile is determined from Heger progenitor model $12 \mathrm{SE}$ as explained in the text. The flat decay portion in the model light curve corresponds to the time during which the initial $\Sigma(r)$ is being redistributed into an accretion disk with a self-consistent radial profile. The lower panel shows the evolution of the locally defined rate of decay $\alpha=-d \log L_{X} / d \log t$. The two quasi-constant portions, $\alpha \simeq 0.4$ and 1.2 , are indicated by the heavy dashed red lines. The long term decay rate $\sim 1.2$ is well-characterized by the analytical solution for electron scattering, gas pressure disks, $\alpha=19 / 16$ (Cannizzo, Lee, \& Goodman 1990).

disk never reaches it. The large dynamic range in disk radii $r_{\text {outer }} / r_{\text {inner }}$ necessitates $\sim 1500-3000$ grid points. In addition, no fresh material is added during a run. Thus the evolution is set entirely by gradients within the disk, unlike the standard Shakura-Sunyaev disk fed at a constant rate in the outer edge which approaches a steady-state $\dot{M}(r)=$ constant with time.

\section{LGRBS}

LGRBs are thought to be associated with the explosions of massive stars (MacFadyen \& Woosley 1999, Woosley \& Heger 2006). The initial failure of the $\mathrm{SN}$ is the reason for the GRB in the collapsar model. The hosts for lGRBs tend to be subluminous, irregular galaxies rich in star formation (Fruchter et al. 2006). If the long-term X-ray light curves of GRBs are indicative of feeding from a fall-back accretion disk, then from Figure 1 we see that no more than $\sim 10^{-4}-10^{-3} M_{\odot}$ of material in the fall-back disk is needed, for nominal assumptions about the efficiencies. In other words, for $\mathrm{a} \sim 10 M_{\odot}$ progenitor, a mass fraction only $\sim 10^{-5}-10^{-4}$ (excluding the $\sim 10 M_{\odot}$ which ends up within

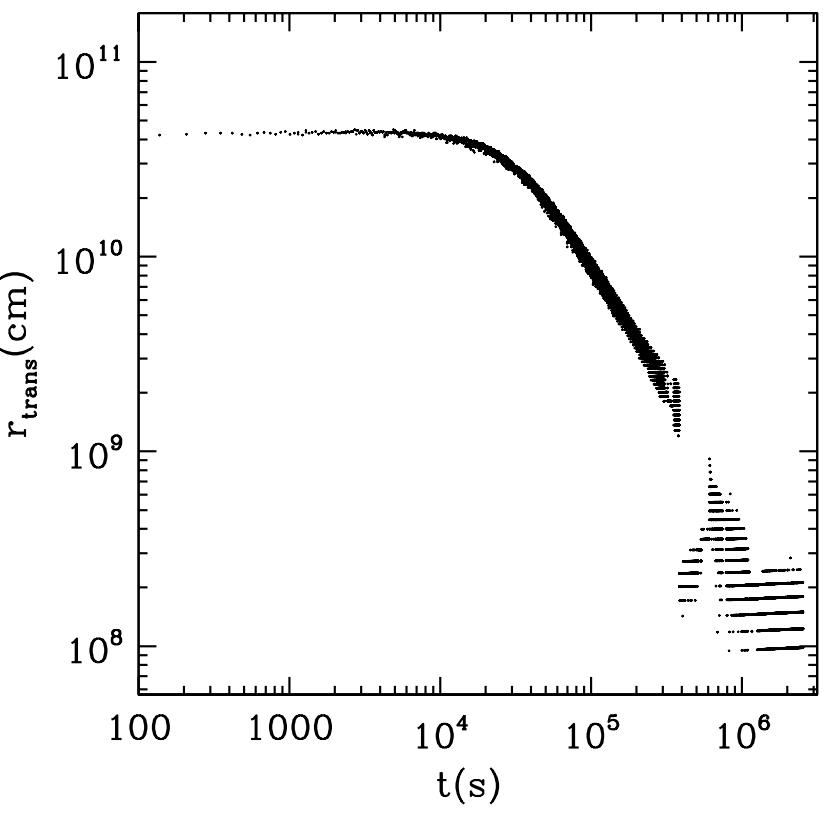

FIG. 4.- The evolution of the boundary between the thick and thin disk states, described in the text, for the run shown in Figures 2 and 3.

$t \lesssim 10-10^{2} \mathrm{~s}$ in the $\mathrm{BH}$ ) is required to survive and persist in the vicinity of the progenitor to power the long-term light curve. If a significant fraction of the mass that tries to ac- 
crete onto the $\mathrm{BH}$ is ejected, the remnant mass around the $\mathrm{BH}$ could be substantially larger. We stress that in this discussion we only include mass accretion beginning in segment II, since segment I is too steep to be accounted for easily in the accretion disk scenario.

Figure 2 shows a light curve for $M_{\mathrm{BH}}=5 M_{\odot}$ and $M_{\text {disk }}=$ $10^{-4} M_{\odot}$, overlaid with XRT data from GRB 060729. The initial $\Sigma(r)$ profile is taken from Heger progenitor ${ }^{7}$ 12SE (Woosley \& Heger 2006) in the following way: The progenitor is collapsed in cylindrical coordinates along the $z$-axis to obtain $\Sigma_{\text {initial }}(r)$, and then scaled down in mass with a radial factor $\propto\left(r / R_{*}\right)$ so that $\int 2 \pi r d r \Sigma_{\text {initial }}(r)$ equals $10^{-4} M_{\odot}$. A radial scaling is also applied so that the integrated specific angular momentum in the final state, taken to represent the disk and therefore to be Keplerian, equals that in the progenitor. This effectively introduces a radial scaling $\sqrt{j_{\text {tot }} / j_{\text {crit }}}$ to the $\Sigma_{\text {initial }}(r)$ distribution. Due to the very long plateau of GRB 060729, we adopted a progenitor model with a large radius. (The value $\log t_{\mathrm{II}}^{*}(\mathrm{~s}) \simeq 4.7$ for GRB 060729 is at the upper end of the distribution of $\log t_{\mathrm{II}}^{*}$ values given in Dainotti et al. 2010.) The lower panel of Figure 2 indicates the local value of the temporal decay index $\alpha$ associated with the model. There are two power law segments evident in the model decay light curve, a brief one $\alpha \simeq 0.4$ between $10^{3}$ and $10^{4} \mathrm{~s}$, and a longer one $\alpha \simeq 1.2$ between $10^{5}$ and $10^{7} \mathrm{~s}$. The latter value $\alpha \simeq 1.2$ is close to that expected from analytical models, 19/16 (Cannizzo, Lee, Goodman 1990). Figure 3 gives the evolution of surface density $\Sigma(r, t)$ and local aspect ratio $h / r$, and Figure 4 indicates the transition point between the slim disk and thin disk states, i.e., $h / r$ dropping below $(3 / 4)^{1 / 2}$.

The steep decay associated with segment I is not accounted for in our model, and may well be due to a separate physical process, such as the accretion of the progenitor core (Kumar et al. 2008b; Lindner et al. 2010, see their Fig. 2). The time scale $t_{\text {II }}$ for the plateau associated with segment II comes out naturally in the models, given the $\sim 10^{11} \mathrm{~cm}$ radius for the progenitor - assuming that the initial fall-back mass distribution roughly traces the envelope of the progenitor (after taking into account the radial scaling factor $\left.\sqrt{j_{\text {tot }} / j_{\text {crit }}}\right)$. The observed X-ray decay for GRB 060729, the GRB which has the been observed for the longest time in X-rays (Grupe et al. 2007, 2010), does not match precisely that of the fall-back disk. There may be various systematic effects that could account for the difference. The most obvious effect, a variable cosmological K-correction concomitant with the fading in Xray flux over a dynamic range of $\sim 6$ decades, cannot play a strong role, as the spectral index does not vary significantly over the long term from its mean value $\beta \simeq 2$. There may be some time-variable physics associated with the X-ray emission from within the jet that could affect either $\epsilon_{\text {net }}$ or $f$ versus time. Our calculations only represent the accretion disk which powers the jet and we take $\dot{\epsilon}_{\text {net }}=\dot{f}=0$ for simplicity. Modeling the jet emission is beyond the scope of this work.

At late times $10^{7} \mathrm{~s}<t<10^{8} \mathrm{~s}$ there is a deviation from the canonical power law decay because $\Sigma\left(r_{\text {outer }}\right)$ drops to the point at which the dwarf nova limit cycle becomes active (indicated by the parallel dashed lines in Figure 3), and a cooling front is launched from the outer edge that instigates a transition from ionized to neutral gas. This quenches the source of accretion onto the central engine. The late time Chandra ob-

\footnotetext{
${ }^{7}$ models available at http: / / $2 \mathrm{sn}$. org/GRB2
}

servations of GRB 060729 (Grupe et al. 2010) appear to coincide with the timing of this drop-off, which may therefore represent a cooling transition front in the outer disk which diminishes the rate of accretion onto the central engine, rather than a jet break.

\section{SGRBS}

SGRBs are thought to be caused by the merger of two neutron stars (Eichler et al. 1989; Paczyński 1991; Narayan, Piran, \& Kumar 2001; Rosswog \& Ramirez-Ruiz 2002). The hosts for sGRBs are indicative of an older population with less active star formation (Fox et al. 2005; Gehrels et al. 2005; Villasenor et al. 2005; Bloom et al. 2006), and also tend to be $\sim 5$ times more spatially extended than those of lGRBs, matching the $\sim 5$ times wider projected spatial distribution of sGRBs vs. lGRBs within their hosts (Fong, Berger, \& Fox 2010). Due to their faintness relative to lGRBs, sGRBs are much less well-studied in X-rays. Indeed, there is only one certifiable example of a sGRB which has a long and wellsampled X-ray light curve, to the extent that a statement can be made as to the presence of a plateau - GRB 051221A.
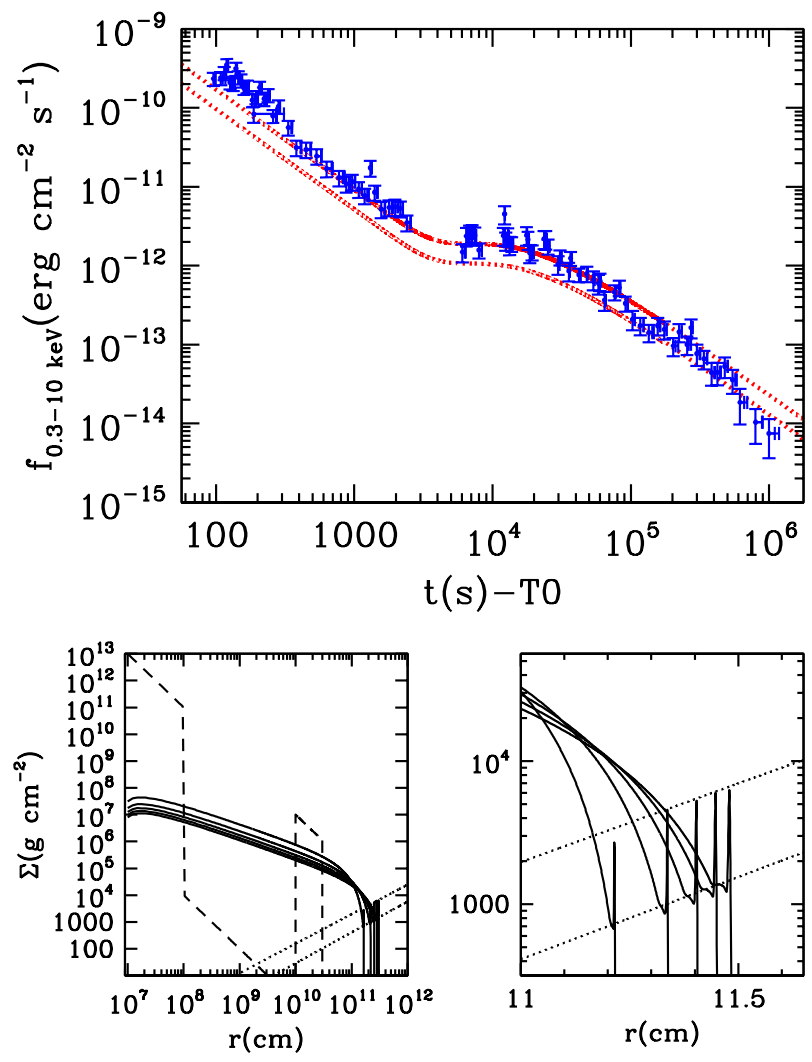

FIG. 5.- Model light curve showing the accretion-derived jet power from the fall-back disk in a sGRB, taking $M_{\mathrm{BH}}=3 M_{\odot}$ and $M_{\text {disk }}=10^{-5} M_{\odot}$ Also shown is the Swift XRT light curve (Evans et al. 2007, 2009) of GRB $051221 \mathrm{~A}$ (in blue). Given the value $4 \pi d_{L}^{2}=1.18 \times 10^{57} \mathrm{~cm}^{2}$ for GRB $051221 \mathrm{~A}(z=0.5465)$, the efficiencies assumed in the two scaled model light curves (in red) to convert from rate of accretion onto the central engine to XRT fluxes are $f \epsilon_{\text {net }}^{-1}=2.4 \times 10^{-2}$ (upper) and $4.3 \times 10^{-2}$ (lower). One sees fairly rapid decay initially due to the presence of a significant amount of accreting material at small radii. The brief shoulder in the light curve is due to the delayed accretion of the spiral arm of ejected NS matter at large radii, motivated by detailed SPH calculations (Rosswog 2007). The bottom panels show the surface density evolution, at equally spaced intervals $\delta t=2 \mathrm{~d}$. The dashed line indicates the initial $\Sigma(r)$ distribution, and the parallel dotted lines indicate the critical values associated with the dwarf nova limit cycle. 
The importance of the outward spreading of the disk formed from the NS-NS merger was clearly demonstrated by Metzger et al. (2008a, see their Fig. 1). The initial radial density profile, and hence $\Sigma(r)$ profile after after angular momentum conservation has vertically compacted the merger remnants into their accretion plane, is of course much more radially condensed than that expected for GRBs. Theoretical guidance on a potential $\Sigma(r)$ profile to begin the calculations, particularly at large radii, is scarce, yet there are indications of small amounts of matter $\sim 10^{-6}-10^{-4} M_{\odot}$ ejected at early times that may have high angular momentum, and therefore circularize at large radii (Rosswog 2007). One potentially important effect we cannot model is the disruption of a portion of the disk by strong nucleosynthesis in the NS supplied material as it expands rapidly to subnuclear densities (Metzger et al. 2008a, 2008b, 2010). Lee, Ramirez-Ruiz, \& LópezCámara (2009) find that strong winds can be launched from the surface of post-merger NS-NS disks, powered by the recombination of free nucleons into $\alpha$-particles.

Figure 5 shows the evolution of a fall-back disk of potential relevance for the aftermath of a NS-NS merger. We take $M_{\mathrm{BH}}=3 M_{\odot}$ and $M_{\mathrm{disk}}=10^{-5} M_{\odot}$. Unlike the much longer evolution shown in Figure 2 for lGRBs, in this case the outer edge of the disk is still freely expanding to larger radii by the end of the run (indicated by the narrow spike in $\Sigma(r)$ between $10^{11} \mathrm{~cm}$ and $3 \times 10^{11} \mathrm{~cm}$ shown in the second lower panel). The brief plateau at $\sim 10^{4} \mathrm{~s}$ results from the ad hoc introduction of the small amount $\sim 10^{-9} M_{\odot}$ of high angular momentum material at the large circularization radius $10^{10} \mathrm{~cm}$. The high efficiencies associated with $\mathrm{BH}$ accretion show the potential for a small amount of material $\ll 1 M_{\odot}$ to have a dramatic effect on the long-term light curves as regards brief plateaus or inflection points.

\section{POTENTIAL APPLICATION TO A DAINOTTI-LIKE RELATION}

In Section 2 we show that the Dainotti relation $L_{\mathrm{II}}^{*} \widetilde{\propto} t^{*-1}$ as originally envisioned may be due to the observational bias against detecting and characterizing faint plateaus. Nevertheless, the accreted mass estimates $\delta M$ inferred using the $L_{\mathrm{II}}^{*}$ and $t_{\mathrm{II}}^{*}$ values from Dainotti et al. (2010) are interesting in the context of this work, and one might legitimately ask what theoretical prediction for the $L_{\mathrm{II}}^{*}\left(t_{\mathrm{II}}^{*}\right)$ relation the fall-back accretion hypothesis would make, given a hypothetical physical constraint in which $\delta M$ were held constant and the initial radius of the fall-back disk were allowed to vary. Figure 6 shows the results of seven runs in which we fix $\delta M=10^{-4} M_{\odot}$ and vary the initial outer radius of the fall back disk. One can see a clear inverse relation between the duration of the plateau $t_{\mathrm{II}}^{*}$ and the luminosity at the end of the plateau $L_{\mathrm{II}}^{*}$. The low $-z$ behavior of $\delta M$ seen in Figure 1, however, indicates that a spread in $\delta M$ may be more realistic, in which case one would not expect to be able to use the theoretical prediction of the $L_{\mathrm{II}}^{*}\left(t_{\mathrm{II}}^{*}\right)$ relation as a useful discriminant for the theory.

\section{DISCUSSION AND CONCLUSION}

We have presented time dependent calculations of the fallback disk scenario to account for the long-term X-ray light curves for GRBs. For lGRBs, an initial radial scale of a stellar radius $\sim 10^{11} \mathrm{~cm}$ gives a natural viscous evolution time of $\sim 10^{4}$ s to redistribute the matter into a quasi-steady disk, roughly consistent with the observed plateau duration. GRB 060729 which we model in the context of a 1GRB had one of the longest plateaus ever observed, thus more generally

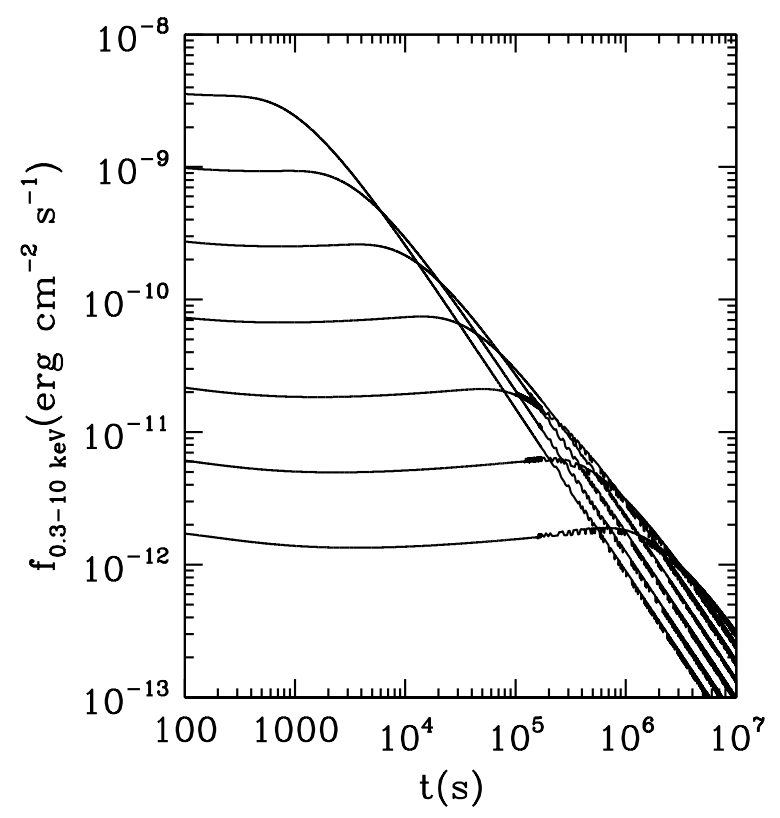

FIG. 6. - Model light curves for lGRB parameters, keeping the initial fall-back disk mass constant at $10^{-4} M_{\odot}$ but varying the initial radius and overall normalization. For the central light curve $r_{0}=7 \times 10^{10} \mathrm{~cm}$. For each successive light curve $r_{0}$ is increased a factor of two going to the right, and decreased a factor of two going to the left. To keep $\delta M$ constant, the overall normalization on $\Sigma(0)$ is varied by an additional factor of 2.5 for each successive run.

we anticipate progenitor radii, or more specifically, circularization radii, given by $\sim \sqrt{j_{\text {tot }} / j_{\text {crit }}} R_{*}$, to lie in the range $\sim 10^{10}-10^{11} \mathrm{~cm}$. The rate of decay for GRB 060729 is close but does not match the models precisely, which may hint at time-variable emission processes that would affect our adopted efficiencies $\epsilon_{\text {net }}$ and $f$. It is interesting to note that our effective values of the efficiencies, accretion plus beaming, required to match the observed X-ray flux levels are comparable between $1 \mathrm{GRB}$ s and sGRBs: $f \epsilon_{\text {net }}^{-1} \simeq 10^{-2}$ for GRB 060729 and $f \epsilon_{\text {net }}^{-1} \simeq 3 \times 10^{-2}$ for GRB 051221A. If the accretion efficiency $\epsilon_{\text {net }}$ is about the same for lGRBs and sGRBs, this may indicate that $\mathrm{sGRBs}$ are less beamed by a factor of $\sim 3$ compared to lGRBs, roughly in line with previous results (Watson et al. 2006, Grupe et al. 2006). This similarity between lGRBs and sGRBs afterglows is not entirely unexpected, given the general similarities in their afterglow properties (Gehrels et al. 2008; Nysewander, Fruchter, \& Pe'er 2009).

The concept of powering the long term X-ray light curve of GRBs by accretion onto the $\mathrm{BH}$ represents a departure from the standard model in which the fading corresponds to the deceleration of a baryonic jet. In the accretion model, the jet itself would be very light, perhaps composed almost exclusively of Poynting flux, and the long term fading would not be due to variations in the Lorentz factor and beaming factor. Recent high fidelity GRMHD calculations of BH accretion support the idea of a high Lorentz factor jet with minimal baryon loading (McKinney \& Narayan 2007ab). The accretion scenario involving fall-back of the progenitor core has been examined in detail by Kumar et al. (2008a, 2008b) and Lindner et al. (2010). Kumar et al. (2008b) argue that constraints may be placed on the density profiles and radii of the progen- 
itor core and envelope, as well as their rotation rates relative to break-up. Lindner et al. (2010) examined the progenitor core fall-back scenario in much greater detail with the adaptive mesh refinement FLASH code, where their calculations are done in cylindrical coordinates. Starting with a Heger progenitor, they follow the fall-back evolution of the progenitor. Their Fig. 2 shows the potential for the model to obtain a steeply decaying light curve as is seen in X-rays for segment I, where $\alpha \simeq 3$ in the Zhang et al. (2006) schematic. In their Discussion, Lindner et al. mention several caveats, such as the lack of nuclear physics in the inner disk, the neglect of the MRI, and the lack of modeling the axial relativistic jet. A more basic concern is simply the range of rate of accretion in comparison to that inferred from observations. Fig. 2 of Lindner et al. shows that if progenitor core fall-back is the correct explanation for segment I in the X-ray light curve, the rate of accretion onto the $\mathrm{BH}$ during this phase varies between about 0.1 and $10^{-5} M_{\odot} \mathrm{s}^{-1}$, whereas from Fig. 2 of this work we see that, for nominal assumptions about the accretion efficiency, the rate inferred from observations on segment I for GRB 060729 varies between about 10 and $3 \times 10^{-3} M_{\odot} \mathrm{yr}^{-1}$, lower by a factor $\sim 10^{5}-10^{6}$ than in Lindner et al. (2010). One does in fact expect for the theoretical accretion rate in this context to be an overestimate, given the potential for outflow, but the discrepancy here seems extreme. There are several possibilities for this discrepancy. The vertical scale shown in Fig. 2 of this work is already super-Eddington by between about one and eight orders of magnitude, and therefore the range in the Lindner et al. calculations would be correspondingly greater. It may be that even highly advective disks cannot accommodate that much accretion onto the $\mathrm{BH}$, and that a significant fraction of the material is blown away before it can accrete. This would have to occur, however, in such a way that it did not interfere with the propagation of the jet. Also, for the shape of the decay light curve calculated by Lindner et al. to correspond to segment I, the ratio of accreted to ejected gas would have to remain constant. If it varied significantly, the accretion-derived luminosity would have a different decay power law. Another possibility is that the Lindner et al. calculations greatly over-estimate the fall-back, in which case a much greater fraction of the progenitor would be ejected on a time scale $\lesssim 10^{3} \mathrm{~s}$. Lindner et al. note that they do not find evidence for the thin disk hypothesized by CG09 as characterizing the fate of the progenitor envelope fall-back. Given the apparent mismatch in accretion rates between their theory and the observations, and the findings of this work, their criticism could have at least two mitigating factors: (1) In Figure 4 we see that for the period covered by the Lindner et al. calculations, namely $t<10^{3} \mathrm{~s}$, a significant fraction of the disk lies within $r_{\text {trans }}$, i.e., the more spatially extended slim disk rather than the thin disk. (2) Given the apparent mismatch between theory and observation for the rates of accretion, the actual densities within the volume formerly occupied by the progenitor may be much less than calculated by Lindner et al. (2010), which would interfere far less with an accretion disk formed from fall-back debris.

At late times Chandra observations indicate a steepening in the rate of decay (Grupe et al. 2010), which appears to be consistent with the onset of a cooling front in the disk. This would be an alternative to the standard jet-break interpretation discussed by Grupe et al. (2010). For sGRBs, the picture is less clear, given that we only have a single well-studied example. We have shown that the presence of even a very small amount of high angular momentum gas $\sim 10^{-9} M_{\odot}$ can give a slight inflection to the X-ray decay, as was observed in GRB 051221A. As for the overall X-ray light curve, segments II and III, if only $\sim 10^{-5}-10^{-4} M_{\odot}$ of gas survives either the hypernova (lGRBs) or NS-NS merger (sGRBs), then the accretion resulting from the ensuing fall-back disk should power a long-term jet.

Lastly, we have shown that the Dainotti relation $L_{\mathrm{II}}^{*} \widetilde{\propto} t^{*-1}$ may be due to an observational bias against detecting and characterizing faint plateaus: the relation is governed by GRBs at $z \gtrsim 1.5$ for which we only detect the upper envelope of a broad distribution. Nevertheless, the existence of an apparent upper limit to the total X-ray energies inferred from the $\mathrm{X}$-ray fluences, and therefore the accreted masses if one assumes accretion onto the central engine as the long term powerhouse for the X-ray flux, is extremely interesting. For nominal values of the accretion efficiency $\epsilon_{\text {net }}$ and the beaming factor $f$, we find an upper limit $\simeq 10^{-4}-10^{-3} M_{\odot}$ for the accreted mass during segments II and III. (The lower end of the distribution of accreted masses, which is partially revealed for GRBs at $z \lesssim 1.5$, may extend down to $\simeq 10^{-8}-10^{-7} M_{\odot}$.) This means that for a progenitor mass $\sim 10 M_{\odot}$, only a maximum mass fraction $\sim 10^{-5}-10^{-4}$ of the progenitor survives in the vicinity of the progenitor to be accreted as a fall-back disk (excluding the $\sim 10 M_{\odot}$ that ends up in the $\mathrm{BH}$ during the prompt emission and subsequent segment I consisting of the steep-decay). This has important ramifications for the energetics associated with the hypernova explosion and subsequent removal of most of the progenitor envelope.

We acknowledge useful conversations with Maria Dainotti, Dirk Grupe, Stephan Rosswog, and Brad Schaefer. This work made use of data supplied by the UK Swift Science Data Centre at the University of Leicester.

\section{REFERENCES}

Abramowicz, M. A., Czerny, B., Lasota, J. P., \& Szuszkiewicz, E. 1988, ApJ, 332,646

Bath, G. T., \& Pringle, J. E. 1981, MNRAS, 194, 967

Bloom, J. S., et al. 2006, ApJ, 638, 354

Cannizzo, J. K. 1993 , ApJ, 419, 318

Cannizzo, J. K. 1998, ApJ, 494, 366

Cannizzo, J. K., \& Gehrels, N. 2009, ApJ, 700, 1047 (CG09)

Cannizzo, J. K., Lee, H.-M., \& Goodman, J. 1990, ApJ, 351, 38

Cannizzo, J. K., \& Reiff, C. M. 1992, ApJ, 385, 87

Cannizzo, J. K., Still, M. D., Howell, S. B., Wood, M. A., \& Smale, A. P. 2010, ApJ, 725, 1393

Chen, W.-X., \& Beloborodov, A. M. 2007, ApJ, 657, 383

Dainotti, M. G., Cardone, V. F., \& Capozziello, S. 2008, MNRAS, 391, L79

Dainotti, M. G., Willingale, R., Capozziello, S., Cardone, V. F., \& Ostrowski, M. 2010, ApJ, 722, L215

Eichler, D., Livio, M., Piran, T., \& Schramm, D. N. 1989, Nature, 340, 126
Evans, P. A., et al. 2007, A\&A, 469, 379

Evans, P. A., et al. 2009, MNRAS, 397, 1177

Fan, Y.-Z., \& Xu, D. 2006, MNRAS, 372, L19

Fong, W., Berger, E., \& Fox, D. B. 2010, ApJ, 708, 9

Fox, D. B., et al. 2005, Nature, 437, 845

Frail, D. A., et al. 2001, ApJ, 562, L55

Fruchter, A. S., et al. 2006, Nature, 441, 463

Gehrels, N., et al. 2004, ApJ, 611, 1005

Gehrels, N., et al. 2005, Nature, 437, 851

Gehrels, N., et al. 2008, ApJ, 689, 1161

Ghisellini, G., Ghirlanda, G., Nava, L., \& Firmani, C. 2007, ApJ, 658, L75

Grupe, D., Burrows, D. N., Patel, S. K., Kouveliotou, C., Zhang, B., Mészáros, P., Wijers, R. A. M., \& Gehrels, N. 2006, ApJ, 653, 462 Grupe, D., et al. 2007, ApJ, 662, 443

Grupe, D., et al. 2010, ApJ, 711, 1008

Kohri, K., Narayan, R., \& Piran, T. 2005, ApJ, 629, 341 
Krolik, J. H., Hawley, J. F. \& Hirose, S. 2007, RevMexAA, 27, 1

Kumar, P., Narayan, R., \& Johnson, J. L. 2008a, MNRAS, 388, 1729

Kumar, P., Narayan, R., \& Johnson, J. L. 2008b, Science, 321, 376

Lasota, J.-P. 2001, New Astron. Rev., 45, 449

Lee, W. H., Ramirez-Ruiz, E., \& López-Cámara, D. 2009, ApJ, 699, L93

Liang, E.-W., Racusin, J. L., Zhang, B., Zhang, B.-B., \& Burrows, D. N. 2008, ApJ, 675, 528

Lindner, C. C., Milosavljević, M., Couch, S. M., \& Kumar, K. 2010, ApJ, 713,800

MacFadyen, A. I., \& Woosley, S. E. 1999, ApJ, 524, 262

McKinney, J. C., \& Narayan, R. 2007a, MNRAS, 375, 513

McKinney, J. C., \& Narayan, R. 2007b, MNRAS, 375, 531

Metzger, B. D., Arcones, A., Quataert, E., \& Martínez-Pinedo, G. 2010, MNRAS, 402, 2771

Metzger, B. D., Piro, A. L., \& Quataert, E. 2008a, MNRAS, 390, 781

Metzger, B. D., Piro, A. L., \& Quataert, E. 2008b, MNRAS, 396, 304

Narayan, R., Piran, T., \& Kumar, P. 2001, ApJ, 557, 949

Nousek, J. A., et al. 2006, ApJ, 642, 389

Nysewander, M., Fruchter, A. S., \& Pe'er, A. 2009, ApJ, 701, 824
Oates, S. R., et al. 2007, MNRAS, 380, 270

Paczyński, B. 1991, Acta Astr., 41, 257

Piran, T. 1978, ApJ, 221, 652

Racusin, J. L., et al. 2008, Nature, 455, 183

Rosswog, S. 2007, MNRAS, 376, L48

Rosswog, S., \& Ramirez-Ruiz, E. 2002, MNRAS, 336, 7

Rowlinson, A., et al. 2010, MNRAS, 409, 531

Sari, R., Piran, T., \& Halpern, J. P. 1999, A\&A, 519, L17

Shakura, N. I., \& Sunyaev, R. A. 1973, A\&A, 24, 337

Smak, J. 1984, Acta Astr., 34, 161

Staff, J., Niebergal, B., \& Ouyed, R. 2008, MNRAS, 391, 178

Toma, K., Ioka, K., Sakamoto, T., \& Nakamura, T. 2007, ApJ, 659, 1420

Villasenor, J. S., et al. 2005, Nature, 437, 855

Watson, D., Hjorth, J., Jakobsson, P., Xu, D., Fynbo, J. P. U., Sollerman, J.,

Thöne, C. C., \& Pedersen, K. 2006, A\&A, 454, L123

Willingale, R., et al. 2007, ApJ, 662, 1093

Woosley, S. E., \& Heger, A. 2006, ApJ, 637, 914

Zhang, B., et al. 2006, ApJ, 642, 354 